\title{
Inefficient Involvement of Insula in Sensorineural Hearing Loss
}

\author{
Xiao-Min Xu' ${ }^{1}$, Yun Jiao' ${ }^{1}$, Tian-Yu Tang ${ }^{1}$, Jian Zhang ${ }^{1}$, Richard Salvi ${ }^{2}$ and Gao-Jun Teng ${ }^{1 *}$ \\ 1 Jiangsu Key Laboratory of Molecular and Functional Imaging, Department of Radiology, Zhongda Hospital, Medical School \\ of Southeast University, Nanjing, China, ${ }^{2}$ Center for Hearing and Deafness, University at Buffalo, Buffalo, NY, United States
}

OPEN ACCESS

Edited by:

Claude Alain,

Rotman Research Institute (RRI),

Canada

Reviewed by:

Jed A. Meltzer,

Baycrest Hospital, Canada

Ming Yang,

Nanjing Medical University, China

*Correspondence:

Gao-Jun Teng

gjteng@seu.edu.cn

Specialty section:

This article was submitted to Auditory Cognitive Neuroscience,

a section of the journal

Frontiers in Neuroscience

Received: 04 November 2018

Accepted: 06 February 2019

Published: 20 February 2019

Citation:

X X X-M, Jiao Y, Tang T-Y, Zhang J, Salvi $R$ and Teng G-J (2019) Inefficient Involvement of Insula in Sensorineural

Hearing Loss.

Front. Neurosci. 13:133. doi: 10.3389/fnins.2019.00133
The insular cortex plays an important role in multimodal sensory processing, audiovisual integration and emotion; however, little is known about how the insula is affected by auditory deprivation due to sensorineural hearing loss (SNHL). To address this issue, we used structural and functional magnetic resonance imaging to determine if the neural activity within the insula and its interregional functional connectivity (FC) was disrupted by SNHL and if these alterations were correlated clinical measures of emotion and cognition. Thirty-five SNHL subjects and 54 Controls enrolled in our study underwent auditory evaluation, neuropsychological assessments, functional and structure MRI, respectively. Twenty five patients and 20 Controls underwent arterial spin labeling scanning. FC of six insula subdivisions were assessed and the FC results were compared to the neuropsychological tests. Interregional connections were also compared among insula-associated networks, including salience network (SN), default mode network (DMN), and central executive network (CEN). Compared to Controls, SNHL subjects demonstrated hyperperfusion in the insula and significantly decreased FC between some insula subdivisions and other brain regions, including thalamus, putamen, precentral gyrus, postcentral gyrus, mid-cingulate cortex, dorsolateral prefrontal cortex, rolandic operculum. Anxiety, depression and cognitive impairments were correlated with FC values. Abnormal interactions among SN, DMN, and CEN were observed in SNHL group. Our result provides support for the "inefficient highorder control" theory of the insula in which the auditory deprivation caused by SNHL contributes to impaired sensory integration and central deficits in emotional and cognitive processing.

\section{Keywords: sensorineural hearing loss, insula, hyperperfusion, functional connectivity, cognition, emotion}

\section{INTRODUCTION}

Sensorineural hearing loss (SNHL), primarily resulting from damage to the sensory hair cells and spiral ganglion neurons, arising from various etiologies, including neurodegenerative disease, noise, and ototoxic drugs (Cox et al., 2014; Wang et al., 2016; Crowson et al., 2017), is the most common sensory disorder affecting roughly one-eighth of the population (Edmiston and Mitchell, 2013; Cunningham and Tucci, 2017). An individual's inability to hear and communicate effectively is associated with a broad range of non-auditory problems such as social isolation, depression, anxiety, and dementia resulting in a reduced quality of life (Bainbridge and Wallhagen, 2014; Basner et al., 2014; Henshaw et al., 2015; Kamil and Lin, 2015; Liu et al., 2016; Zhang et al., 2017a). The emotional and cognitive 
disorders associated with SNHL likely results from disturbances in neural networks outside the classical auditory pathway that integrate external and internal sensory information required for cognitive or emotional processes.

The insula, located deep within the lateral sulcus, has been implicated in numerous functions including emotion, awareness, cognition, motor control, and sensory processing (Craig, 2009; Bestelmeyer et al., 2014). Insula neurons have been found to respond directly to acoustic stimuli in a singlecell recording study (Bieser, 1998). Micarelli et al. (2017) highlighted hypoperfusion in the insula and auditory cortex in idiopathic sudden SNHL patients, reflecting a "freezing" behavior when auditory deprivation occurred abruptly. The function and structure of the insula were found to be impaired in patients with tinnitus and unilateral hearing loss using resting-state or task-based MRI approaches (Yang et al., 2014). Lesions of the insula or disconnections with the auditory cortex often result in auditory agnosia and musical anhedonia (Sihvonen et al., 2016). Damage to the insula can induce hyperacusis, a condition in which sounds are perceived as extremely loud (Boucher et al., 2015). Moreover, the insula has numerous connections with frontal cortex, cingulate cortex and amygdala which contribute to emotional, cognitive and other high level processes (Pandya et al., 1971; Hoistad and Barbas, 2008; Baur et al., 2013; Nomi et al., 2016), as well as neuropsychiatric disorders (Namkung et al., 2017), including depression, anxiety, and bipolar disorder (Stein et al., 2007; Liu et al., 2010; Hulvershorn et al., 2012). These preceding results suggest that the insula is a key structure of modulating acoustic information and could contribute to SNHL-associated psychiatric symptoms.

To elaborate the role of the insula in patients with SNHL, we first used voxel-based morphometry (VBM) to calculate the volume of the insula, then used arterial spin labeling (ASL) and fMRI to determine whether SNHL disrupted the activity and perfusion within the insula, altered the functional connectivity (FC) between the insula and other brain regions. We also examined the interactions among the salience network (SN), default mode network (DMN), and central executive network (CEN), due to the fact that the insula is a major component of $\mathrm{SN}$, which plays an important role in switching CEN and DMN. Because of known regional differences (Deen et al., 2011), our analysis focused on six regions, the left and right ventral anterior insula (vAI), dorsal AI (dAI), and posterior insula (PI). To evaluate the potential significance of our finding, the functional changes in the insula associated with SNHL were correlated with clinical measures of neuropsychological function.

\section{MATERIALS AND METHODS}

\section{Participants}

Eighty-nine participants were recruited from the ENT department of local hospitals or the local community for this study, 35 subjects with a mean age of $56.1 \pm 8.6$ years and a mean education of $10.9 \pm 3.0$ years had long-term bilateral SNHL and 54 subjects were age-, gender-, and education level-matched healthy controls with clinical normal hearing. Both groups underwent T1-weighted image scan (T1WI), BOLD sequences and a series of neuropsychological tests. Only 25 SNHL subjects (10 males and 15 females) and 20 Controls ( 4 males and 16 females) agreed to complete the next arterial spin labeling (ASL) scan (see Table 1 and Figure 1). All subjects were randomly assigned using double-blinded principles for further analysis.

Participants were all right-handed and had at least 6 years of education, with ages ranging from 38 to 65 years. Patients meeting the diagnostic criteria of SNHL $>1$ year. Participants were excluded if they (1) suffered from tinnitus, hyperacusis, acoustic neuroma, Meniere's diseases, head trauma, visual loss, severe heart diseases or MRI contraindications; (2) had a history of head surgery, stroke, cognitive impairment or other serious physical and psychotic diseases; (3) presented with drug dependence, alcohol or substance abuse.

The study was approved by the ethics committee of Zhongda Hospital, Southeast University (2016ZDSYLL031.0). Written informed consent in accordance with the Declaration of Helsinki was obtained from all subjects.

\section{Auditory Evaluation}

Clinical pure tone audiometry (PTA) test (Chen et al., 2018) was performed at $0.125,0.25,0.5,1,2,4,8 \mathrm{kHz}$ by a trained otolaryngologist with 12 years of work experience from the ENT Department of Zhongda Hospital using a GSI-61 audiometer. In

TABLE 1 | Clinical characteristics of SNHL and the control groups and local measurements of the left and right insula.

\begin{tabular}{|c|c|c|c|}
\hline Characteristics & SNHL $(n=35)$ & Control $(n=54)$ & $P$-value \\
\hline \multicolumn{4}{|l|}{ Clinical measurements } \\
\hline Gender (male/female) & $22 / 13$ & $34 / 20$ & $0.992^{\mathrm{a}}$ \\
\hline Age (years) & $56.1 \pm 8.6$ & $53.5 \pm 7.9$ & $0.153^{b}$ \\
\hline Education (years) & $10.9 \pm 3.0$ & $12.4 \pm 5.2$ & $0.136^{\mathrm{b}}$ \\
\hline Duration (years) & $6.9 \pm 6.7$ & - & - \\
\hline \multicolumn{4}{|c|}{ Neuropsychological tests } \\
\hline MMSE & $29.6 \pm 0.8$ & $29.7 \pm 0.5$ & $0.352^{b}$ \\
\hline SDMT & $33.4 \pm 12.2$ & $40.7 \pm 8.9$ & $0.003^{b}$ \\
\hline AVLT-5 min & $5.7 \pm 2.5$ & $6.6 \pm 2.0$ & $0.070^{b}$ \\
\hline AVLT-20 min & $5.6 \pm 2.5$ & $6.5 \pm 2.2$ & $0.079^{b}$ \\
\hline SAS & $35.8 \pm 7.1$ & $30.9 \pm 5.5$ & $<0.001^{\mathrm{b}}$ \\
\hline HAMD & $6.1 \pm 4.0$ & $4.4 \pm 2.9$ & $0.025^{b}$ \\
\hline \multicolumn{4}{|l|}{ Volume } \\
\hline $\begin{array}{l}\text { Relative volume of left } \\
\text { insula }\end{array}$ & $0.406 \pm 0.007$ & $0.411 \pm 0.006$ & $0.592^{b}$ \\
\hline $\begin{array}{l}\text { Relative volume of right } \\
\text { insula }\end{array}$ & $0.405 \pm 0.007$ & $0.409 \pm 0.006$ & $0.612^{b}$ \\
\hline Perfusion & SNHL $(n=25)$ & Control $(n=20)$ & $P$-value \\
\hline $\begin{array}{l}\text { Mean CBF of left insula } \\
(\mathrm{ml} / 100 \mathrm{~g} / \mathrm{min})\end{array}$ & $71.610 \pm 2.847$ & $61.100 \pm 2.975$ & $0.013^{b}$ \\
\hline $\begin{array}{l}\text { Mean CBF of right } \\
\text { insula (ml/100 g/min) }\end{array}$ & $78.780 \pm 3.109$ & $67.230 \pm 3.429$ & $0.018^{b}$ \\
\hline
\end{tabular}

Data are represented as mean \pm standard deviation. "Chi-square test ${ }^{b}$ Independent-sample t-test. SNHL, sensorineural hearing loss; MMSE, Mini Mental State Exam; SDMT, Symbol Digit Modalities Test; AVLT, Auditory Verbal Learning Test; SAS, Self-Rating Anxiety Scale; HAMD, Hamilton Depression Scale; CBF, cerebral blood flow. 


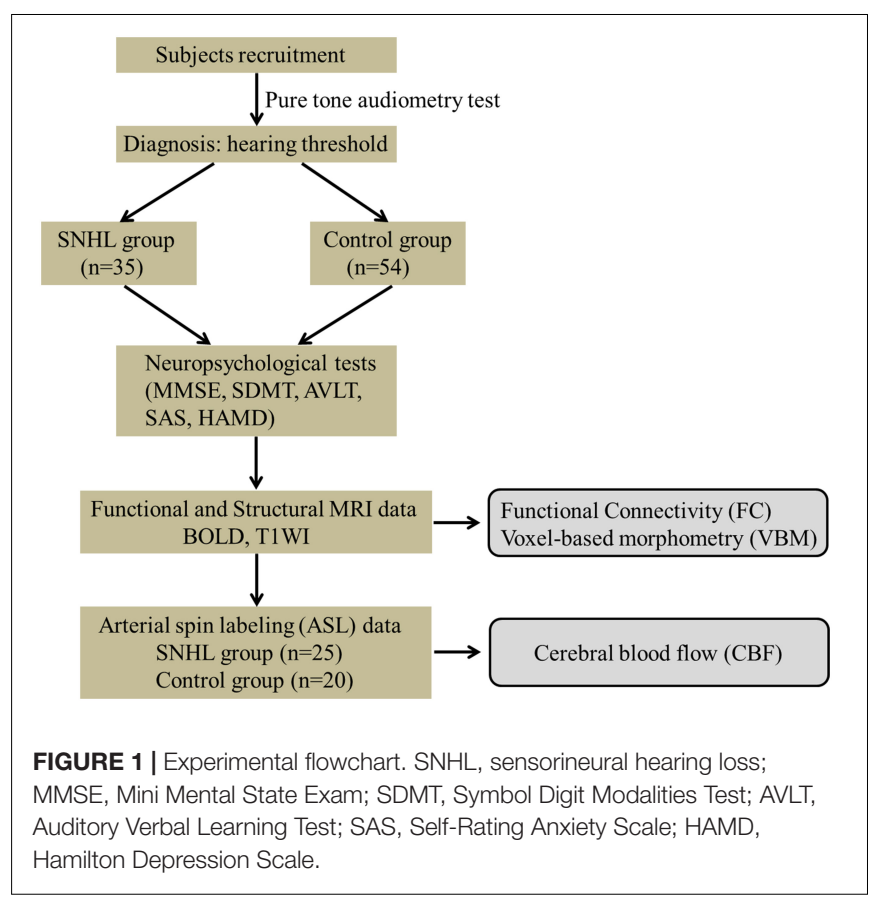

the control group, the mean PTA across the seven frequencies was $<25 \mathrm{~dB}$ HL (Lin et al., 2011), while the mean PTA of SNHL across the seven frequencies was $>30 \mathrm{~dB}$ HL. According to global burden of disease (GBD) 2013, hearing loss has been ranked into five severity levels, as moderate, 35-49 dB; moderately severe, 50-64 dB; severe, 65-79 dB; profound, 80-94 dB; and complete $>95 \mathrm{~dB}$.

\section{Neuropsychological Assessment}

All subjects underwent a battery of neuropsychological tests prior to the MRI scans and these tests were carried out by a professional psychiatrist with 6 years of work experience to provide a measure of their cognitive and mental status. General cognition was established by the Mini Mental State Exam (MMSE) (Galea and Woodward, 2005), the Symbol Digit Modalities Test (SDMT) (Patel et al., 2017), and the Auditory Verbal Learning Test (AVLT) (Hawkins et al., 2004). Depression and anxiety status were assessed using the Hamilton Depression Scale (HAMD) (Maier et al., 1985) and the Self-Rating Anxiety Scale (SAS) (Zung, 1971).

\section{Brain Imaging Acquisition}

MRI studies were performed in a Siemens 3.0 Tesla scanner using a homogeneous birdcage head coil. Subjects lay supine and were required to close their eyes, stay awake and avoid thinking specific thoughts while in the scanner. We used ear plugs and earphones to attenuate scanner noise and a head cushion to reduce head motion. High-resolution 3-dimensional T1WI scans were acquired using a spoiled gradient-echo sequence [repetition time $(\mathrm{TR})=1900 \mathrm{~ms}$, echo time $(\mathrm{TE})=2.48 \mathrm{~ms}$, flip angle $(\mathrm{FA})=90^{\circ}$, field of view $(\mathrm{FOV})=256 \mathrm{~mm} \times 256 \mathrm{~mm}$, acquisition matrix $=256 \times 256$, slices $=176$, section thickness $=1.0 \mathrm{~mm}]$. A gradient-echo-planar imaging sequence was set up to obtain functional images $(\mathrm{TR}=2000 \mathrm{~ms}, \mathrm{TE}=25 \mathrm{~ms}, 36$ slices, section thickness $=4.0 \mathrm{~mm}, \mathrm{FA}=90^{\circ}, \mathrm{FOV}=240 \mathrm{~mm} \times 240 \mathrm{~mm}$, acquisition matrix $=64 \times 64)$. Subsequent ASL perfusion MR was performed using a Siemens product pulsed-ASL (pASL) PICORE Q2T sequence $(\mathrm{TR}=4000 \mathrm{~ms}, \mathrm{TE}=12 \mathrm{~ms}, 27$ slices, thickness $=4 \mathrm{~mm} ; \mathrm{FA}=90^{\circ} ;$ matrix $=64 \times 64$; $\mathrm{FOV}=220 \mathrm{~mm} \times 220 \mathrm{~mm})$.

\section{Data Processing Protocol Functional Data}

Two experienced radiologists inspected all image data. As described in previous studies (Chen et al., 2014; Cui et al., 2014), data analysis was conducted using DPARBI toolbox ${ }^{1}$, which is based on DPARSF (version 4.3), SPM 12 ${ }^{2}$, and REST ${ }^{3}$. After removing the first 10 time points, the remaining 230 times points were corrected for slice timing, realignment of head motion, segmentation and normalization to the non-linear Montreal Neurological Institute (MNI) template (resampling to $3 \mathrm{~mm} \times 3 \mathrm{~mm} \times 3 \mathrm{~mm}$ voxels). Then regressed six motion parameters, white matter, and CSF signals. Afterward, the images were smoothed using a $6 \mathrm{~mm}$ full-width half-maximum (FWHM) Gaussian kernel. No one was excluded from this study because of head motion $>2.0 \mathrm{~mm}$ translation or $>2.0^{\circ}$ rotation in any direction.

\section{Structure Data}

Individual structural images were analyzed with the DARTELVBM method (Colloby et al., 2011, 2014) in the following order: (1) segment the MRI images into the gray matter (GM), white matter and cerebrospinal fluid standard unified segmentation model in SPM 12; (2) construct GM templates from the entire image dataset (Ashburner, 2007) to generate tissue probability maps in MNI space; (3) perform non-linear warping of segmented images to match the MNI space to DARTEL templates; (4) modulate the relative GM volume following spatial normalization; (5) smooth data with a $6 \mathrm{~mm}$ FWHM Gaussian kernel.

\section{Perfusion Data}

Similar to (Zhang et al., 2018), SPM 12 and ASLtbx (Wang et al., 2008) were used to process pASL data. First, motion corrections were performed to eliminate spurious motion artifacts. The raw ASL images were then high pass filtered to retain the higher frequency band. Then, the ASL images were co-registered to the T1 images and spatially smoothed with a $6 \mathrm{~mm}$ FWHM Gaussian kernel. After subsequent pairwise control/label image subtraction and cerebral blood flow (CBF) quantification, rejection of $\mathrm{CBF}$ outliers, mean $\mathrm{CBF}$ maps were created and registered into the MNI space using the transformation obtained from structural images.

\section{Statistical Analysis}

Demographic, clinical variables and scores of neuropsychological performance were compared by independent-sample t-tests

\footnotetext{
${ }^{1}$ http://rfmri.org/DPABI

${ }^{2}$ http://www.fil.ion.ucl.ac.uk/spm

${ }^{3}$ http://www.restfmri.net
} 
using SPSS software (Version 18.0, United States). Chi-square tests were used to compare categorical variables (e.g., gender). $P$-values $<0.05$ were considered statistically significant.

Using the Anatomical Automatic Labeling (AAL) atlas, we selected the left and right insula as two ROIs. We extracted the relative volume and mean CBF values of the two ROIs and identified changes in structure and perfusion caused by SNHL (two-sample $t$-test, $p<0.05$ ). Consistent with previous research (Zamorano et al., 2017; Zhang et al., 2017b), bilateral vAI, dAI and PI subdivisions were used as seeds in the whole-brain FC analysis (see Table 2 for the coordinates of six ROIs). The resulting FC maps were transformed using Fisher's $\mathrm{z}$ to yield normally distributed data and the data averaged for each subject. False discovery rate (FDR) correction was used to correct for multiple comparisons with a corrected $p<0.001$. To examine changes in FC caused by SNHL, a partial correlation analysis with some covariates (including age, gender and education level) between $z$-values of the FC results and the clinical measurements were performed using SPSS software (Version 18.0, United States). Statistical significance was set a $p<0.05$.

The three large-scale networks, CEN, DMN, and SN, are believed to be involved in psychiatric disorders (Menon, 2011) and the insula is a key node of SN. Therefore, we investigated the changes in the interregional relations of these three networks following SNHL. The nodes representing CEN and DMN were selected according to previous research (Moran et al., 2013): CEN: dorsolateral prefrontal cortex (DLPFC), inferior parietal lobule (IPL), and Caudate; DMN: medial prefrontal cortex (MPFC), posterior cingulate cortex (PCC), lateral parietal cortex (LP), and parahippocampal gyrus (PHG). ROI-wise FC analysis was conducted among CEN, DMN, and SN, with FDR correction for multiple comparison (corrected $p<0.05$ ). All above analysis on the MRI data were conducted using the age, gender and education level as covariance.

\section{RESULTS}

\section{Clinical Characteristics and Local Measurements of the Insula}

The demographic and clinical characteristics of SNHL and Controls are summarized in Table 1. Both groups were wellmatched in terms of gender, age and education level. SNHL subjects performed significantly worse that Controls on SDMT

TABLE 2 | Name and coordinates of insula subdivisions ( $r=6 \mathrm{~mm})$.

\begin{tabular}{lllccc}
\hline Hemisphere & Subdivision & Abbreviation & \multicolumn{3}{c}{ Coordinate } \\
\cline { 4 - 6 } & & & $\boldsymbol{X}$ & $\boldsymbol{Y}$ & $\boldsymbol{Z}$ \\
\hline \multirow{2}{*}{ Left } & Ventral anterior insula & $\mathrm{vAl}$ & -33 & 13 & -7 \\
& Dorsal anterior insula & $\mathrm{dAl}$ & -38 & 6 & 2 \\
& Posterior insula & $\mathrm{Pl}$ & -38 & -6 & 5 \\
\multirow{3}{*}{ Right } & Ventral anterior insula & $\mathrm{vAl}$ & 32 & 10 & -6 \\
& Dorsal anterior insula & $\mathrm{dAl}$ & 35 & 7 & 3 \\
& Posterior insula & $\mathrm{Pl}$ & 35 & -11 & 6
\end{tabular}

$(p<0.003)$, SAS $(p<0.001)$ and HAMD $(p=0.025)$ tests. Figure 2 illustrates the differences in hearing thresholds between the SNHL group and Controls at $0.125,0.25,0.5,1,2,4$, and $8 \mathrm{kHz}$. Thresholds in the SNHL group were significantly higher in the SNHL group than Controls $(p<0.001)$, especially at the high frequencies ( 4 and $8 \mathrm{kHz}$ ).

There was no significant difference in the relative volume of bilateral insula between two groups (Table 1). However, $\mathrm{CBF}$ values of the left and right insula were significantly greater in SNHL group compared to Controls, indicating the hyperperfusion of the insula after auditory deprivation.

\section{Voxel-Wise Functional Connectivity of Insula Subdivisions}

Using six insula subdivisions as ROIs, FC analysis revealed an extensive reduction of connectivity between the insula and other brain regions $(p<0.001$, FDR corrected, minimum cluster $=50$ ). Compared to Controls, the SNHL group showed a significant reduction in FC between the left vAI and the bilateral thalamus and right precentral gyrus. Additionally, the SNHL group displayed weakened FC between left dAI and the right thalamus, putamen, DLPFC, precentral gyrus, postcentral gyrus and mid-cingulate cortex. Moreover, the SNHL group demonstrated decreased FC between the right $\mathrm{dAI}$ and right rolandic operculum. Finally, the SNHL group demonstrated decreased FC between the right PI and right thalamus, as well as right precentral gyrus (Figure 3 and Table 3).

\section{Functional Connectivity and Clinical Features}

Partial correlation analysis with some covariates (age, gender, and education level) identified significant relationships between clinical characteristics and FC data. In the SNHL group, FC of the left dAI and right DLPFC was negatively correlated with SAS scores $(r=-0.489, p=0.005$, Figure 4A) and positively correlated with SDMT sores $(r=0.410, p=0.020$, Figure $4 \mathrm{~B})$. FC in the

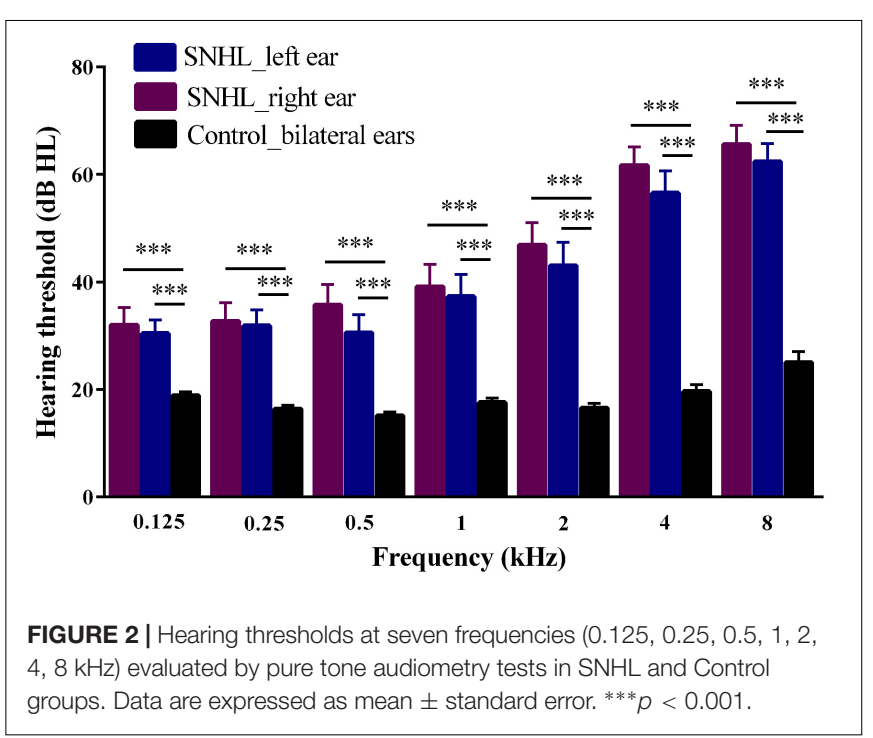


left $\mathrm{dAI}$ and the right mid-cingulate cortex FC was negatively correlated with HAMD scores $(r=-0.402, p=0.022$, Figure 4C). However, no correlation survived after multiple comparison.

\section{Network Abnormalities in CEN, DMN, and SN}

To determine if SNHL affected the 18 core regions involved in the CEN, DMN, and SN, we employed a ROI-wise interregional connectivity analysis to identify significant changes (Figure 5). SNHL significantly weakened interactions among these three networks as follows. The left vAI showed decreased interregional connectivity with the left LP while the right vAI showed decreased connections with left LP and right PCC. And the left dAI showed reduced connectivity with bilateral DLPFC, PCC and right PCC. Meanwhile, SNHL reduced the connectivity between left PI and right PCC, as well as the connections between right PI and bilateral PCC and left LP ( $p<0.05$, FDR corrected).

\section{DISCUSSION}

To our knowledge, this is the first study to determine if SNHL disrupts the function and connective of the insula and interregional connectivity using a multimodal neuroimaging approach. We found significant hyperperfusion in left and right insula in subjects with SNHL and widespread decreased neuronal synchronizations between insula subdivisions and other brain regions. Several of these FC changes were correlated with altered performance on neuropsychological metrics of emotion and cognition. Furthermore, SNHL altered the interregional connections among CEN, DMN, and SN. Taken together, our findings provide new insights on the role of insula in SNHLrelated neuropsychiatric deficits.

\section{SNHL and Structure}

Our VBM analysis failed to identify significant volume differences in the insula between groups, consistent with absence
A

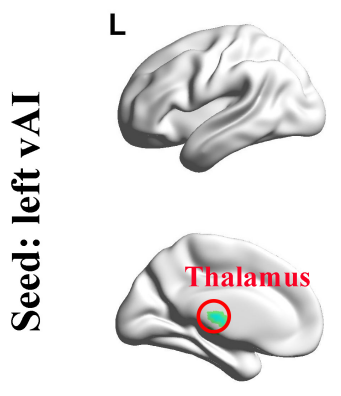

C

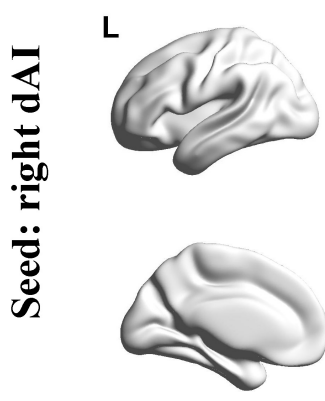

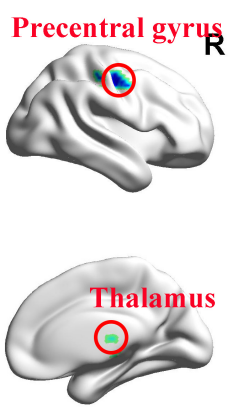

D

\section{B}
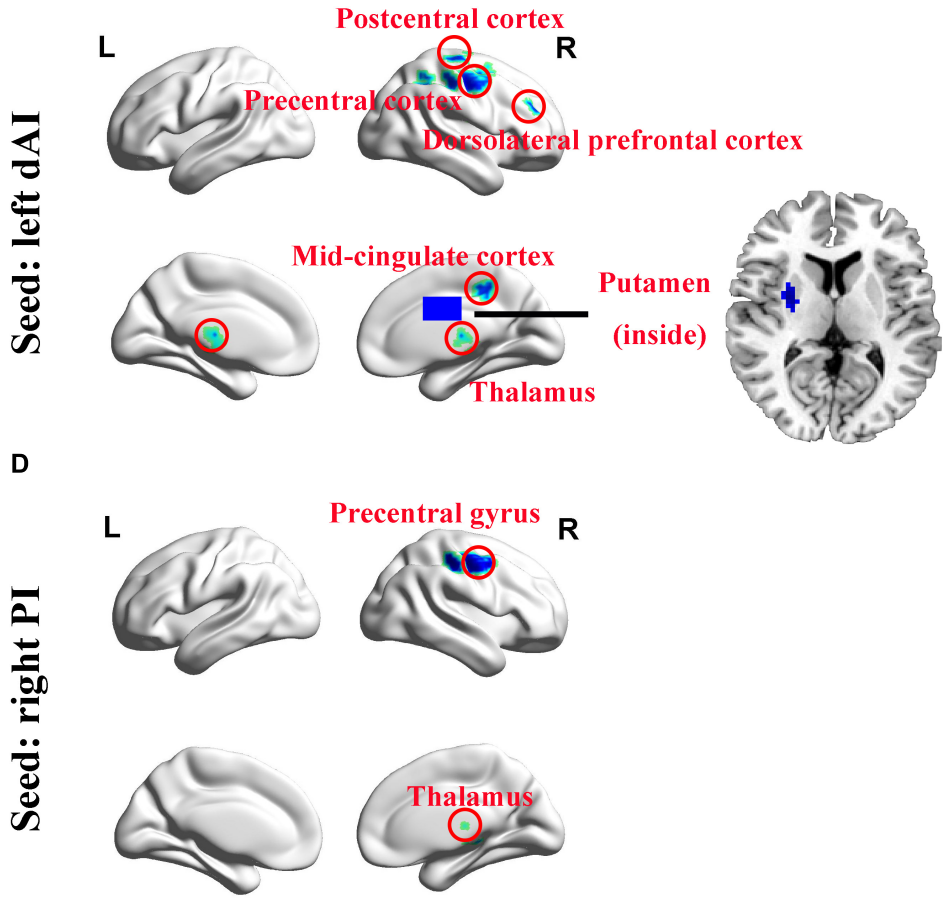

$\mathbf{R}$

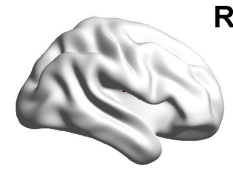

Rolandic operculum
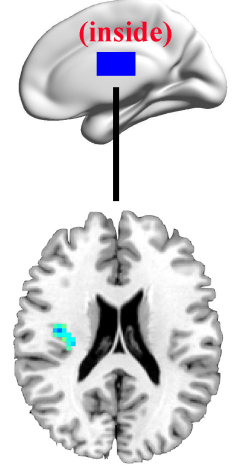

FIGURE 3 | Whole-brain voxel-wise functional connectivity patterns of the insula subdivisions ( $p<0.001$, false discovery rate corrected). (A) Comparing with Controls, functional connectivity was significantly decreased between left vAl and bilateral thalamus and right precentral gyrus in SNHL subjects. (B) The left dAI showed significant decreased functional connectivity with right thalamus, putamen, mid-cingulate cortex, precentral gyrus, postcentral gyrus, and DLPFC. (C) The right dAl showed decreased connectivity with right rolandic operculum. (D) The right PI showed weakened connections with right thalamus and precentral gyrus. SNHL, sensorineural hearing loss; vAl, ventral anterior insula; dAI, dorsal anterior insula; PI, posterior insula; DLPFC, dorsolateral prefrontal cortex; L, left; R, right. 
TABLE 3 | Decreased functional connectivity of the insula subdivisions in SNHL subjects comparing with Controls.

\begin{tabular}{|c|c|c|c|c|c|c|c|}
\hline \multirow[t]{2}{*}{$\begin{array}{l}\text { Brain } \\
\text { region }\end{array}$} & \multirow[t]{2}{*}{ BA } & \multirow[t]{2}{*}{ Side } & \multicolumn{3}{|c|}{$\begin{array}{c}\text { MNI } \\
\text { coordinate }\end{array}$} & \multirow[t]{2}{*}{ Peak $t$-value } & \multirow[t]{2}{*}{$\begin{array}{c}\text { Cluster } \\
\text { size }\end{array}$} \\
\hline & & & $x$ & $Y$ & $Z$ & & \\
\hline \multicolumn{8}{|c|}{ Functional connectivity of left vAl } \\
\hline Thalamus & - & $R$ & 12 & -22 & 9 & -7.2482 & 102 \\
\hline Thalamus & - & $L$ & -6 & -18 & 6 & -6.736 & 57 \\
\hline Precentral & 6 & $\mathrm{R}$ & 42 & -12 & 45 & -6.3206 & 68 \\
\hline
\end{tabular}

gyrus

\section{Functional connectivity of left dAl}

\begin{tabular}{|c|c|c|c|c|c|c|c|}
\hline Thalamus & - & $\mathrm{R}$ & 6 & -21 & 9 & -7.6285 & 298 \\
\hline Putamen & 48 & $\mathrm{R}$ & 30 & -3 & 9 & -5.8026 & 81 \\
\hline $\begin{array}{l}\text { Dorsolateral } \\
\text { prefrontal } \\
\text { cortex }\end{array}$ & 46 & $\mathrm{R}$ & 42 & 42 & 27 & -5.3125 & 53 \\
\hline $\begin{array}{l}\text { Precentral } \\
\text { gyrus }\end{array}$ & 6 & $\mathrm{R}$ & 42 & -12 & 45 & -6.227 & 303 \\
\hline $\begin{array}{l}\text { Mid- } \\
\text { cingulate } \\
\text { cortex }\end{array}$ & - & $\mathrm{R}$ & 15 & -36 & 39 & -5.522 & 53 \\
\hline $\begin{array}{l}\text { Postcentral } \\
\text { gyrus }\end{array}$ & 6 & $\mathrm{R}$ & 27 & -27 & 66 & -5.3037 & 62 \\
\hline \multicolumn{8}{|c|}{ Functional connectivity of right dAl } \\
\hline $\begin{array}{l}\text { Rolandic } \\
\text { operculum }\end{array}$ & 48 & $\mathrm{R}$ & 39 & -12 & 21 & -5.8744 & 56 \\
\hline \multicolumn{8}{|c|}{ Functional connectivity of right PI } \\
\hline Thalamus & - & $\mathrm{L}$ & 9 & -21 & 9 & -6.4883 & 108 \\
\hline $\begin{array}{l}\text { Precentral } \\
\text { gyrus }\end{array}$ & 6 & $\mathrm{R}$ & 39 & -12 & 45 & -6.4082 & \\
\hline
\end{tabular}

The threshold was set at $p<0.001$, false discovery rate correction. BA, Brodmann area; vAl, ventral anterior insula; $d A I$, dorsal anterior insula; $P I$, posterior insula.

of major structural changes in the insula of prelingual and postlingual hearing loss (Shibata, 2007; Hribar et al., 2014; Shiell and Zatorre, 2017). However, a few studies (Allen et al., 2008) reported structural alterations in the human insula from congenital deafness; in these cases sign language increased GM volume of left PI compared to normal hearing subjects. Compared to hearing non-signers, hearing signers, and deaf signers exhibited a significant increase in the size of the right insula, which was attributed to lip-reading and articulation, rather than auditory input itself (MacSweeney et al., 2001). In contrast, the SNHL patients in our study all had acquired postlingual hearing loss and lived with normal hearing families. Thus, the most likely explanation for the lack of structural changes in our study was the lack of sign language history. We did not ask our controls and SNHL whether they were signers and cannot address the question about the effects of articulation. All things being equal, it is likely that signing was equally distributed across the SNHL and the control group, making it difficult to identify an articulatory effect in our data.

\section{Perfusion}

Pulsed-ASL is a non-invasive and sensitive imaging technique for assessing CBF without using radioactive sources or contrast agents (Williams et al., 1992; Detre and Alsop, 1999). In comparison to PET, pASL has superior spatial resolution and sensitivity, therefore it has been widely used to measure $\mathrm{CBF}$ in cerebrovascular and psychiatric disorders (Cui et al., 2017). Our observation of heighted CBF values in the insula was independent of cortical atrophy, as no volume difference were observed between SNHL subjects and Controls in the insula. Lee et al. (2001) reported hypoperfusion in the primary cortex and auditory-association cortex of prelingually deaf patients; the hypoperfusion was positively correlated with degree of hearing improvement after cochlear implantation. Prior PET reports from subject with postlingual deafness $(n=7)$ (Okuda et al., 2013) and idiopathic sudden
A

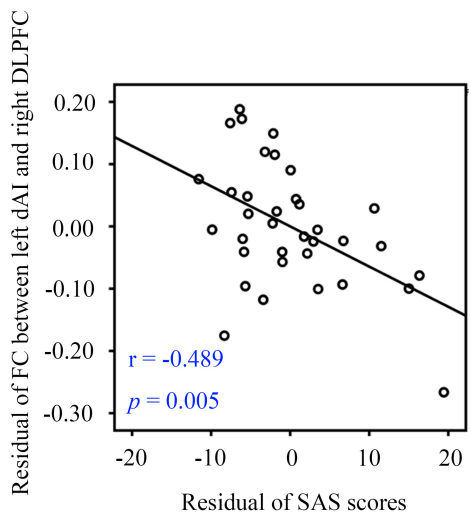

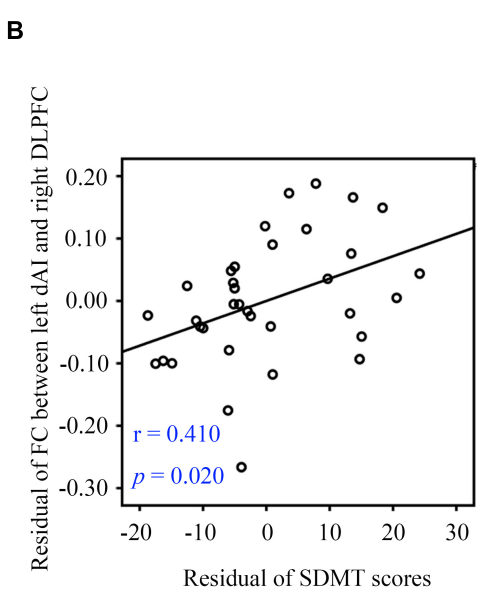

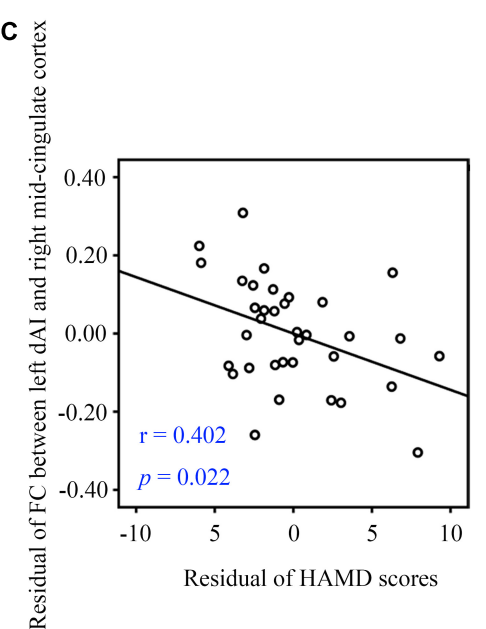

FIGURE 4 | Partial correlations between fMRI data and clinical measurements. (A) The left dAI-right DLPFC connectivity showed a negative correlation with SAS scores. (B) The left dAI-right DLPFC connectivity showed a positive correlation with SDMT scores. (C) The left dAI-right mid-cingulate cortex connectivity showed a negative correlation with HAMD scores. SNHL, sensorineural hearing loss; dAl, dorsal anterior insula; SDMT, Symbol Digit Modalities Test; SAS, Self-Rating Anxiety Scale; HAMD, Hamilton Depression Scale; DLPFC, dorsolateral prefrontal cortex. 
A

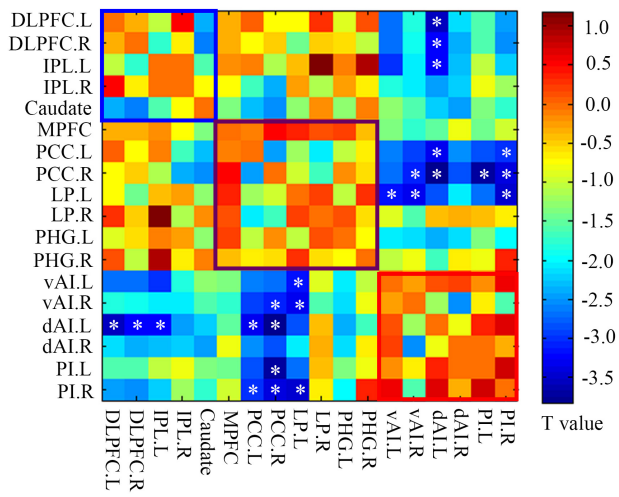

B

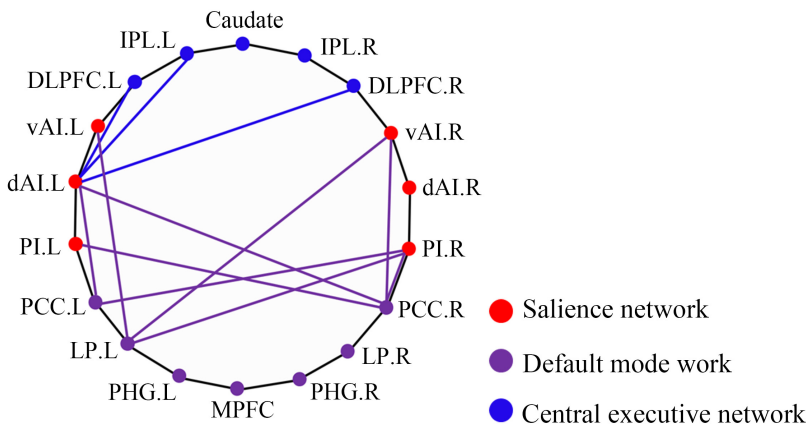

C

\begin{tabular}{ccccc}
\hline ROI & R & \multicolumn{3}{c}{ Coordinate } \\
& $(\mathbf{m m})$ & $\mathbf{X}$ & $\mathbf{Y}$ & $\mathbf{Z}$ \\
\hline Central executive network (CEN) & & & & \\
Left dorsolateral prefrontal cortex (DLPFC) & 6 & 44 & 45 & 9 \\
Right dorsolateral prefrontal cortex (DLPFC) & 6 & -44 & 45 & 9 \\
Left inferior parietal lobule(IPL) & 6 & 60 & -42 & 33 \\
Right inferior parietal lobule(IPL) & 6 & -60 & -42 & 33 \\
$\quad$ Caudate & 10 & 0 & 3 & 42 \\
Default mode network(DMN) & & & & \\
Medial prefrontal cortex (MPFC) & 10 & 0 & 60 & 6 \\
Left posterior cingulate cortex (PCC) & 6 & 9 & -51 & 27 \\
Right posterior cingulate cortex (PCC) & 6 & -9 & -51 & 27 \\
Left lateral parietal cortex (LP) & 6 & 51 & -55 & 27 \\
Right lateral parietal cortex (LP) & 6 & -51 & -55 & 27 \\
Left parahippocampal gyrus (PHG) & 6 & 30 & -15 & -18 \\
Right parahippocampal gyrus (PHG) & 6 & -30 & -15 & -18 \\
\hline
\end{tabular}

FIGURE 5 | ROI-wise interregional connectivity analysis among salience network, central executive network and default mode network across all participants $(p<0.05$, false discovery rate corrected). (A) Colored matrix indicating $t$-values of interactions among salience network, central executive network and default mode network. (B) Significant difference in interregional functional connectivity between SNHL and Controls. (C) Detailed ROI coordinates extracted from central executive network and default mode network. SNHL, sensorineural hearing loss. ${ }^{*} p<0.05$.

SNHL patients $(n=14)$ (Micarelli et al., 2017) indicated lower glucose metabolism in the temporal lobe and insula attributed to the absence of sensory input. The difference between our insula results and these earlier reports may be related to the types of hearing loss (i.e., unilateral vs. bilateral, genetic factors), duration of deafness, small sample size, and other factors such as cognitive and emotional differences as illustrated by worse scores on SDMT, SAS, and HAMD in our SNHL subjects. Others have noted a relationship between the perfusion of the insula and anxiety (Andreescu et al., 2011). Moreover, it has been reported that depression could activate the insula and amygdala, propounding an "anxious-misery" dimension (Kennedy et al., 2001; Xekardaki et al., 2015). Thus, the increased perfusion in the insula might be associated with SNHL-induced anxiety and depression in our subject sample. We speculate that the CBF could be a confirmatory candidate biomarker for diagnosis since it is tightly linked to regional metabolism and neural activity.

\section{Regional Insula Functional Connectivity Changes}

Most insula subdivisions in our SNHL subjects showed decreased connectivity with the thalamus which might reflect a change in sensory-emotional integration (Groenewegen and Berendse, 1994). The thalamus receives numerous sensory inputs and then relays this information to associated higherorder primary cortical areas (Lee, 2015). Reward stimuli can increase the thalamus-to-insula connectivity (Cho et al., 2013) whereas negative stimuli, such as difficulties hearing due to partial deafness, could have the opposite effect thereby reducing connectivity. It is conceivable that reduced incoming auditory information results in weakened connectivity between the thalamus and insula. Additionally, we observed reduced connections between the left $\mathrm{dAI}$ and right putamen while the putamen has been found to connect to the thalamus and functioned in cognition decline, anxiety and depression (Park et al., 2017; Luo et al., 2018). 
Traditionally, the dAI and vAI are activated by cognition and emotion task, respectively (Nomi et al., 2016). However, this cognition-emotion dichotomy has been challenged recently (Uddin et al., 2014). Our data showed reduced couplings between the left dAI and right mid-cingulate cortex in SNHL subjects, as well as the negative correlation with HAMD scores. The mid-cingulate cortex is not only linked to cognitive processing, including decision making, attention and salience (Dosenbach et al., 2007; Rushworth et al., 2007), but also homeostasis and emotion, and it has become a therapy target for intractable mood, anxiety, and pain disorders (Tolomeo et al., 2016). Patients with treatment-resistant depression or refractory pain sometimes receive bilateral cingulotomy (including AI) (Steele et al., 2008; Shackman et al., 2011). Primate and human studies (Mufson and Mesulam, 1982; Taylor et al., 2009) provide evidence for functional connections between the AI and midcingulate cortex, as well as FC between the entire insula and mid-cingulate cortex. In addition, thalamus-insula- midcingulate cortex projection tracts have been found in animals (Hatanaka et al., 2003), suggesting that functional connections between the $\mathrm{dAI}$ and mid-cingulate cortex might contribute to neuropsychiatric disorders.

Reduced fluorodeoxyglucose (FDG) uptake has been observed in the insula, precentral gyrus and postcentral gyrus within $72 \mathrm{~h}$ following sudden SNHL (Micarelli et al., 2017). Although the postcentral gyrus has often been linked to somatosensory processing, pain and olfaction (Bedny et al., 2011; Grabski et al., 2012), studies have demonstrated greater activation in this region in speech rhythm, auditory oddball and articulation imagery tasks (Geiser et al., 2008; Job et al., 2012; Tian et al., 2016), suggesting its involvement in speech perception. The volume of the precentral gyrus is reduced in schizophrenia (Zhou et al., 2005), indicating that the precentral gyrus (part of the primary motor cortex) is involved in motor-related cognitive functioning (Georgopoulos, 2000); these observations are relevant to poorer SDMT performance in our SNHL subjects. Moreover, the right dAI showed decreased connectivity with rolandic operculum as it together covers with the insula and was reported hyperactivity in tinnitus subjects following acute acoustic trauma (Job et al., 2012).

Some insula subdivisions showed decreased connectivity with the DLPFC, PCC, and LP using voxel-wise and ROIwise methods, suggesting the involvement of the DMN and CEN in SNHL-induced auditory deprivation. A meta-analysis showed that several brain regions overlap with the DMN and CEN play an important role in processing multiple cognitive signals (Dosenbach et al., 2006), while acquiring normal sensory information supports cognition functioning. SNHL disrupts the auditory system, leading to the disruptions of the $\mathrm{SN}$, resulting in imbalances between the DMN and CEN. Chand et al. (2017), also found abnormal interactions among the SN, DMN, and CEN in subjects with mild cognitive impairments. Recent research suggest that that depression and anxiety are associated with alterations in the DMN, expanding the role of DMN in emotion processing (Vicentini et al., 2017), as FC of left dAI-right DLPFC not only showed positive correlation with SDMT performance, but also negative correlation with
SAS scores. Combing the hyperperfusion in the insula, we used an "inefficient high-order control" theory to illustrate this phenomenon.

\section{Limitation}

There were several limitations to our research. First, it is reported that human left and right insula occupy a total volume of around $10-20 \mathrm{~cm}^{3}$ (Bauernfeind et al., 2013) while we employed 6-mm diameter spherical ROIs as seeds, further research with independent component analysis could be used to extract the insula subregions. Second, the relative small sample of subjects likely reduced the statistical power, particularly for the correlation analysis. A follow-up study with a larger dataset might help to elucidate more subtle effects in the future. Another limitation was the range and duration of hearing loss in our study. Repeating aspects of this study using subjects with more severe and/or longer duration hearing loss might identify other functional and structural changes associated with SNHL.

\section{CONCLUSION}

In conclusion, this study underscores the potential contribution of disrupted neural processing in the insula resulting from SNHL and its potential relationships with neuropsychiatric disorders, suggesting that it could be a candidate biomarker for auditory deprivation related neural deficits and a future target for therapy.

\section{AUTHOR CONTRIBUTIONS}

X-MX collected the fMRI data, performed the analysis, and wrote the manuscript. YJ and T-YT contributed to fMRI data analysis and discussion. JZ helped with data collection. RS reviewed and revised the manuscript. G-JT designed the MRI experiments and contributed to the manuscript revision.

\section{FUNDING}

This work was supported by the National Natural Science Foundation of China (81520108015), the Fundamental Research Funds for the Central Universities (2242018k30004), and Clinical Innovation Center of Medical Imaging and Interventional Radiology (YXZXA2016005).

\section{ACKNOWLEDGMENTS}

We thank Xu Feng and Zhi-Chun Huang, ENT Department, Affiliated Zhongda Hospital of Southeast University, for their assistance with data collection. We also thank Jing Zhao, ENT Department, Affiliated Zhongda Hospital of Southeast University, for her assistance with auditory evaluation. 


\section{REFERENCES}

Allen, J. S., Emmorey, K., Bruss, J., and Damasio, H. (2008). Morphology of the insula in relation to hearing status and sign language experience. J. Neurosci. 28, 11900-11905. doi: 10.1523/JNEUROSCI.3141-08.2008

Andreescu, C., Gross, J. J., Lenze, E., Edelman, K. D., Snyder, S., Tanase, C., et al. (2011). Altered cerebral blood flow patterns associated with pathologic worry in the elderly. Depress. Anxiety 28, 202-209. doi: 10.1002/da.20799

Ashburner, J. (2007). A fast diffeomorphic image registration algorithm. Neuroimage 38, 95-113. doi: 10.1016/j.neuroimage.2007.07.007

Bainbridge, K. E., and Wallhagen, M. I. (2014). Hearing loss in an aging American population: extent, impact, and management. Annu. Rev. Public Health 35 139-152. doi: 10.1146/annurev-publhealth-032013-182510

Basner, M., Babisch, W., Davis, A., Brink, M., Clark, C., Janssen, S., et al. (2014). Auditory and non-auditory effects of noise on health. Lancet 383, 1325-1332. doi: 10.1016/S0140-6736(13)61613-X.

Bauernfeind, A. L., de Sousa, A. A., Avasthi, T., Dobson, S. D., Raghanti, M. A., Lewandowski, A. H., et al. (2013). A volumetric comparison of the insular cortex and its subregions in primates. J. Hum. Evol. 64, 263-279. doi: 10.1016/j. jhevol.2012.12.003

Baur, V., Hanggi, J., Langer, N., and Jancke, L. (2013). Resting-state functional and structural connectivity within an insula-amygdala route specifically index state and trait anxiety. Biol. Psychiatry 73, 85-92. doi: 10.1016/j.biopsych.2012.06.003

Bedny, M., Pascual-Leone, A., Dodell-Feder, D., Fedorenko, E., and Saxe, R. (2011). Language processing in the occipital cortex of congenitally blind adults. Proc. Natl. Acad. Sci. U.S.A. 108, 4429-4434. doi: 10.1073/pnas.10148 18108

Bestelmeyer, P. E., Maurage, P., Rouger, J., Latinus, M., and Belin, P. (2014) Adaptation to vocal expressions reveals multistep perception of auditory emotion. J. Neurosci. 34, 8098-8105. doi: 10.1523/JNEUROSCI.4820-13.2014

Bieser, A. (1998). Processing of twitter-call fundamental frequencies in insula and auditory cortex of squirrel monkeys. Exp. Brain Res. 122, 139-148. doi: 10.1007/ s002210050501

Boucher, O., Turgeon, C., Champoux, S., Menard, L., Rouleau, I., Lassonde, M., et al. (2015). Hyperacusis following unilateral damage to the insular cortex: a three-case report. Brain Res. 1606, 102-112. doi: 10.1016/j.brainres.2015.02.030

Chand, G. B., Wu, J., Hajjar, I., and Qiu, D. (2017). Interactions of the salience network and its subsystems with the default-mode and the central-executive networks in normal aging and mild cognitive impairment. Brain Connect. 7 , 401-412. doi: 10.1089/brain.2017.0509

Chen, Y. C., Chen, H., Jiang, L., Bo, F., Xu, J. J., Mao, C. N., et al. (2018). Presbycusis disrupts spontaneous activity revealed by resting-state functional MRI. Front. Behav. Neurosci. 12:44. doi: 10.3389/fnbeh.2018.00044

Chen, Y. C., Jiao, Y., Cui, Y., Shang, S. A., Ding, J., Feng, Y., et al. (2014). Aberrant brain functional connectivity related to insulin resistance in type 2 diabetes: a resting-state fMRI study. Diabetes Care 37, 1689-1696. doi: 10.2337/dc132127

Cho, Y. T., Fromm, S., Guyer, A. E., Detloff, A., Pine, D. S., Fudge, J. L., et al. (2013). Nucleus accumbens, thalamus and insula connectivity during incentive anticipation in typical adults and adolescents. Neuroimage 66, 508-521. doi: 10.1016/j.neuroimage.2012.10.013

Colloby, S. J., Firbank, M. J., Vasudev, A., Parry, S. W., Thomas, A. J., and O’Brien, J. T. (2011). Cortical thickness and VBM-DARTEL in late-life depression. J. Affect. Disord. 133, 158-164. doi: 10.1016/j.jad.2011.04.010

Colloby, S. J., O’Brien, J. T., and Taylor, J. P. (2014). Patterns of cerebellar volume loss in dementia with Lewy bodies and Alzheimer's disease: a VBM-DARTEL study. Psychiatry Res. Neuroimag. 223, 187-191. doi: 10.1016/j.pscychresns. 2014.06.006

Cox, R. M., Johnson, J. A., and Xu, J. (2014). Impact of advanced hearing aid technology on speech understanding for older listeners with mild to moderate, adult-onset, sensorineural hearing loss. Gerontology 60, 557-568. doi: 10.1159/ 000362547

Craig, A. D. (2009). How do you feel - now? The anterior insula and human awareness. Nat. Rev. Neurosci. 10, 59-70. doi: 10.1038/nrn2555

Crowson, M. G., Hertzano, R., and Tucci, D. L. (2017). Emerging therapies for sensorineural hearing loss. Otol. Neurotol. 38, 792-803. doi: 10.1097/MAO. 0000000000001427
Cui, Y., Jiao, Y., Chen, Y. C., Wang, K., Gao, B., Wen, S., et al. (2014). Altered spontaneous brain activity in type 2 diabetes: a restingstate functional MRI study. Diabetes 63, 749-760. doi: 10.2337/db130519

Cui, Y., Liang, X., Gu, H., Hu, Y., Zhao, Z., Yang, X. Y., et al. (2017). Cerebral perfusion alterations in type 2 diabetes and its relation to insulin resistance and cognitive dysfunction. Brain Imaging Behav. 11, 1248-1257. doi: 10.1007/ s11682-016-9583-9

Cunningham, L. L., and Tucci, D. L. (2017). Hearing loss in adults. N. Engl. J. Med. 377, 2465-2473. doi: 10.1056/NEJMra1616601

Deen, B., Pitskel, N. B., and Pelphrey, K. A. (2011). Three systems of insular functional connectivity identified with cluster analysis. Cereb. Cortex 21, 14981506. doi: 10.1093/cercor/bhq186

Detre, J. A., and Alsop, D. C. (1999). Perfusion magnetic resonance imaging with continuous arterial spin labeling: methods and clinical applications in the central nervous system. Eur. J. Radiol. 30, 115-124. doi: 10.1016/S0720048X(99)00050-9

Dosenbach, N. U., Fair, D. A., Miezin, F. M., Cohen, A. L., Wenger, K. K., Dosenbach, R. A., et al. (2007). Distinct brain networks for adaptive and stable task control in humans. Proc. Natl. Acad. Sci. U.S.A. 104, 11073-11078. doi: 10.1073/pnas.0704320104

Dosenbach, N. U., Visscher, K. M., Palmer, E. D., Miezin, F. M., Wenger, K. K., Kang, H. C., et al. (2006). A core system for the implementation of task sets. Neuron 50, 799-812. doi: 10.1016/j.neuron.2006.04.031

Edmiston, R., and Mitchell, C. (2013). Hearing loss in adults. BMJ 346:f2496. doi: $10.1136 /$ bmj.f2496.

Galea, M., and Woodward, M. (2005). Mini-Mental State Examination (MMSE). Aust. J. Physiother. 51:198. doi: 10.1016/S0004-9514(05)70 034-9

Geiser, E., Zaehle, T., Jancke, L., and Meyer, M. (2008). The neural correlate of speech rhythm as evidenced by metrical speech processing. J. Cogn. Neurosci. 20, 541-552. doi: 10.1162/jocn.2008.20029

Georgopoulos, A. P. (2000). Neural aspects of cognitive motor control. Curr. Opin. Neurobiol. 10, 238-241. doi: 10.1016/S0959-4388(00)00072-6

Grabski, K., Lamalle, L., and Sato, M. (2012). Somatosensory-motor adaptation of orofacial actions in posterior parietal and ventral premotor cortices. PLoS One 7:e49117. doi: 10.1371/journal.pone.0049117

Groenewegen, H. J., and Berendse, H. W. (1994). The specificity of the nonspecific midline and intralaminar thalamic nuclei. Trends Neurosci. 17, 52-57 doi: 10. 1016/0166-2236(94)90074-4

Hatanaka, N., Tokuno, H., Hamada, I., Inase, M., Ito, Y., Imanishi, M., et al. (2003). Thalamocortical and intracortical connections of monkey cingulate motor areas. J. Comp. Neurol. 462, 121-138. doi: 10.1002/cne.10720

Hawkins, K. A., Dean, D., and Pearlson, G. D. (2004). Alternative forms of the rey auditory verbal learning test: a review. Behav. Neurol. 15, 99-107. doi: 10.1155/2004/940191

Henshaw, H., Sharkey, L., Crowe, D., and Ferguson, M. (2015). Research priorities for mild-to-moderate hearing loss in adults. Lancet 386, 2140-2141. doi: 10. 1016/S0140-6736(15)01048-X

Hoistad, M., and Barbas, H. (2008). Sequence of information processing for emotions through pathways linking temporal and insular cortices with the amygdala. Neuroimage 40, 1016-1033. doi: 10.1016/j.neuroimage.2007. 12.043

Hribar, M., Suput, D., Carvalho, A. A., Battelino, S., and Vovk, A. (2014). Structural alterations of brain grey and white matter in early deaf adults. Hear Res. 318, 1-10. doi: 10.1016/j.heares.2014. 09.008

Hulvershorn, L. A., Karne, H., Gunn, A. D., Hartwick, S. L., Wang, Y., Hummer, T. A., et al. (2012). Neural activation during facial emotion processing in unmedicated bipolar depression, euthymia, and mania. Biol. Psychiatry 71, 603-610. doi: 10.1016/j.biopsych.2011.10.038

Job, A., Pons, Y., Lamalle, L., Jaillard, A., Buck, K., Segebarth, C., et al. (2012). Abnormal cortical sensorimotor activity during "Target" sound detection in subjects with acute acoustic trauma sequelae: an fMRI study. Brain Behav. 2, 187-199. doi: 10.1002/brb3.21

Kamil, R. J., and Lin, F. R. (2015). The effects of hearing impairment in older adults on communication partners: a systematic review. J. Am. Acad. Audiol. 26, 155-182. doi: 10.3766/jaaa.26.2.6 
Kennedy, B. L., Schwab, J. J., Morris, R. L., and Beldia, G. (2001). Assessment of state and trait anxiety in subjects with anxiety and depressive disorders. Psychiatr. Q. 72, 263-276. doi: 10.1023/A:1010305200087

Lee, C. C. (2015). Exploring functions for the non-lemniscal auditory thalamus. Front. Neural Cir. 9:69. doi: 10.3389/fncir.2015.00069

Lee, D. S., Lee, J. S., Oh, S. H., Kim, S. K., Kim, J. W., Chung, J. K., et al. (2001). Cross-modal plasticity and cochlear implants. Nature 409, 149-150 doi: $10.1038 / 35051653$

Lin, F. R., Thorpe, R., Gordon-Salant, S., and Ferrucci, L. (2011). Hearing loss prevalence and risk factors among older adults in the United States. J. Gerontol. A Biol. Sci. Med. Sci. 66, 582-590. doi: 10.1093/gerona/glr002

Liu, L., Shen, P., He, T., Chang, Y., Shi, L., Tao, S., et al. (2016). Noise induced hearing loss impairs spatial learning/memory and hippocampal neurogenesis in mice. Sci. Rep. 6:20374. doi: 10.1038/srep20374

Liu, Z., Xu, C., Xu, Y., Wang, Y., Zhao, B., Lv, Y., et al. (2010). Decreased regional homogeneity in insula and cerebellum: a resting-state fMRI study in patients with major depression and subjects at high risk for major depression. Psychiatry Res. 182, 211-215. doi: 10.1016/j.pscychresns.2010.03.004

Luo, L., Becker, B., Zheng, X., Zhao, Z., Xu, X., Zhou, F., et al. (2018). A dimensional approach to determine common and specific neurofunctional markers for depression and social anxiety during emotional face processing. Hum. Brain Mapp. 39, 758-771. doi: 10.1002/hbm.23880

MacSweeney, M., Campbell, R., Calvert, G. A., McGuire, P. K., David, A. S., Suckling, J., et al. (2001). Dispersed activation in the left temporal cortex for speech-reading in congenitally deaf people. Proc. Biol. Sci. 268, 451-457. doi: $10.1098 / \mathrm{rspb} .2000 .0393$

Maier, W., Philipp, M., and Gerken, A. (1985). [Dimensions of the Hamilton Depression Scale. Factor analysis studies]. Eur. Arch. Psychiatry Neurol. Sci. 234, 417-422. doi: 10.1007/BF00386061

Menon, V. (2011). Large-scale brain networks and psychopathology: a unifying triple network model. Trends Cogn. Sci. 15, 483-506. doi: 10.1016/j.tics.2011. 08.003

Micarelli, A., Chiaravalloti, A., Viziano, A., Danieli, R., Schillaci, O., and Alessandrini, M. (2017). Early cortical metabolic rearrangement related to clinical data in idiopathic sudden sensorineural hearing loss. Hear Res. 350, 91-99. doi: 10.1016/j.heares.2017.04.011

Moran, L. V., Tagamets, M. A., Sampath, H., O’Donnell, A., Stein, E. A. Kochunov, P., et al. (2013). Disruption of anterior insula modulation of largescale brain networks in schizophrenia. Biol. Psychiatry 74, 467-474. doi: 10 1016/j.biopsych.2013.02.029

Mufson, E. J., and Mesulam, M. M. (1982). Insula of the old world monkey. II afferent cortical input and comments on the claustrum. J. Comp. Neurol. 212, 23-37. doi: $10.1002 /$ cne. 902120103

Namkung, H., Kim, S., and Sawa, A. (2017). The insula: an underestimated brain area in clinical neuroscience, psychiatry, and neurology. Trends Neurosci. 40, 200-207. doi: 10.1016/j.tins.2017.02.002

Nomi, J. S., Farrant, K., Damaraju, E., Rachakonda, S., Calhoun, V. D., and Uddin, L. Q. (2016). Dynamic functional network connectivity reveals unique and overlapping profiles of insula subdivisions. Hum. Brain Mapp. 37, 1770-1787. doi: $10.1002 / \mathrm{hbm} .23135$

Okuda, T., Nagamachi, S., Ushisako, Y., and Tono, T. (2013). Glucose metabolism in the primary auditory cortex of postlingually deaf patients: an FDGPET study. ORL J. Otorhinolaryngol. Relat. Spec. 75, 342-349. doi: 10.1159/ 000357474

Pandya, D. N., Karol, E. A., and Heilbronn, D. (1971). The topographical distribution of interhemispheric projections in the corpus callosum of the rhesus monkey. Brain Res. 32, 31-43. doi: 10.1016/0006-8993(71)90 153-3

Park, I. H., Lee, B. C., Kim, J. J., Kim, J. I., and Koo, M. S. (2017). Effort-based reinforcement processing and functional connectivity underlying amotivation in medicated patients with depression and schizophrenia. J. Neurosci. 37, 4370-4380. doi: 10.1523/JNEUROSCI.2524-16.2017

Patel, V. P., Walker, L. A. S., and Feinstein, A. (2017). Deconstructing the symbol digit modalities test in multiple sclerosis: the role of memory. Mult. Scler. Relat. Disord. 17, 184-189. doi: 10.1016/j.msard.2017.08.006

Rushworth, M. F., Buckley, M. J., Behrens, T. E., Walton, M. E., and Bannerman, D. M. (2007). Functional organization of the medial frontal cortex. Curr. Opin. Neurobiol. 17, 220-227. doi: 10.1016/j.conb.2007.03.001
Shackman, A. J., Salomons, T. V., Slagter, H. A., Fox, A. S., Winter, J. J., and Davidson, R. J. (2011). The integration of negative affect, pain and cognitive control in the cingulate cortex. Nat. Rev. Neurosci. 12, 154-167. doi: 10.1038/ nrn2994

Shibata, D. K. (2007). Differences in brain structure in deaf persons on MR imaging studied with voxel-based morphometry. AJNR Am. J. Neuroradiol. 28, 243-249.

Shiell, M. M., and Zatorre, R. J. (2017). White matter structure in the right planum temporale region correlates with visual motion detection thresholds in deaf people. Hear Res. 343, 64-71. doi: 10.1016/j.heares.2016. 06.011

Sihvonen, A. J., Ripolles, P., Leo, V., Rodriguez-Fornells, A., Soinila, S., and Sarkamo, T. (2016). Neural basis of acquired amusia and its recovery after stroke. J. Neurosci. 36, 8872-8881. doi: 10.1523/JNEUROSCI.0709-16.2016

Steele, J. D., Christmas, D., Eljamel, M. S., and Matthews, K. (2008). Anterior cingulotomy for major depression: clinical outcome and relationship to lesion characteristics. Biol. Psychiatry 63, 670-677. doi: 10.1016/j.biopsych.2007.07.019

Stein, M. B., Simmons, A. N., Feinstein, J. S., and Paulus, M. P. (2007). Increased amygdala and insula activation during emotion processing in anxiety-prone subjects. Am. J. Psychiatry 164, 318-327. doi: 10.1176/ajp.2007.164.2.318

Taylor, K. S., Seminowicz, D. A., and Davis, K. D. (2009). Two systems of resting state connectivity between the insula and cingulate cortex. Human Brain Mapp. 30, 2731-2745. doi: 10.1002/hbm.20705

Tian, X., Zarate, J. M., and Poeppel, D. (2016). Mental imagery of speech implicates two mechanisms of perceptual reactivation. Cortex 77, 1-12. doi: 10.1016/j. cortex.2016.01.002

Tolomeo, S., Christmas, D., Jentzsch, I., Johnston, B., Sprengelmeyer, R., Matthews, K., et al. (2016). A causal role for the anterior mid-cingulate cortex in negative affect and cognitive control. Brain 139(Pt 6), 1844-1854. doi: 10.1093/ brain/aww069

Uddin, L. Q., Kinnison, J., Pessoa, L., and Anderson, M. L. (2014). Beyond the tripartite cognition-emotion-interoception model of the human insular cortex. J. Cogn. Neurosci. 26, 16-27. doi: 10.1162/jocn_a_00462

Vicentini, J. E., Weiler, M., Almeida, S. R. M., de Campos, B. M., Valler, L., and Li, L. M. (2017). Depression and anxiety symptoms are associated to disruption of default mode network in subacute ischemic stroke. Brain Imaging Behav. 11, 1571-1580. doi: 10.1007/s11682-016-9605-7

Wang, S., Yang, M., Du, S., Yang, J., Liu, B., Gorriz, J. M., et al. (2016). Wavelet entropy and directed acyclic graph support vector machine for detection of patients with unilateral hearing loss in MRI scanning. Front. Comput. Neurosci. 10:106. doi: 10.3389/fncom.2016.00106

Wang, Z., Aguirre, G. K., Rao, H., Wang, J., Fernandez-Seara, M. A., Childress, A. R., et al. (2008). Empirical optimization of ASL data analysis using an ASL data processing toolbox: ASLtbx. Magnet. Reson. Imaging 26, 261-269. doi: 10.1016/j.mri.2007.07.003

Williams, D. S., Detre, J. A., Leigh, J. S., and Koretsky, A. P. (1992). Magneticresonance-imaging of perfusion using spin inversion of arterial water. Proc. Natl. Acad. Sci. U.S.A. 89, 212-216. doi: 10.1073/pnas.89.1.212

Xekardaki, A., Rodriguez, C., Montandon, M. L., Toma, S., Tombeur, E., Herrmann, F. R., et al. (2015). Arterial spin labeling may contribute to the prediction of cognitive deterioration in healthy elderly individuals. Radiology 274, 490-499. doi: 10.1148/radiol.14140680

Yang, M., Chen, H. J., Liu, B., Huang, Z. C., Feng, Y., Li, J., et al. (2014). Brain structural and functional alterations in patients with unilateral hearing loss. Hear Res. 316, 37-43. doi: 10.1016/j.heares.2014.07.006

Zamorano, A. M., Cifre, I., Montoya, P., Riquelme, I., and Kleber, B. (2017). Insulabased networks in professional musicians: Evidence for increased functional connectivity during resting state fMRI. Hum. Brain Mapp. 38, 4834-4849. doi: $10.1002 / \mathrm{hbm} .23682$

Zhang, Y., Nayak, D. R., Yang, M., Yuan, T. F., Liu, B., Lu, H., et al. (2017a). Detection of unilateral hearing loss by stationary wavelet entropy. CNS Neurol. Disord. Drug Targets 16, 122-128. doi: 10.2174/187152731566616102611 5046

Zhang, Y., Yang, Y., Bian, R., Yin, Y., Hou, Z., Yue, Y., et al. (2017b). Group cognitive behavior therapy reversed insula subregions functional connectivity in asthmatic patients. Front. Aging Neurosci. 9:105. doi: 10.3389/fnagi.2017. 00105 
Zhang, Y., Yang, Y., Wang, Z., Bian, R., Jiang, W., Yin, Y., et al. (2018). Altered regional cerebral blood flow of right cerebellum posterior lobe in asthmatic patients with or without depressive symptoms. Front. Psychiatry 9:225. doi: 10.3389/fpsyt.2018.00225

Zhou, S. Y., Suzuki, M., Hagino, H., Takahashi, T., Kawasaki, Y., Matsui, M., et al. (2005). Volumetric analysis of sulci/gyri-defined in vivo frontal lobe regions in schizophrenia: Precentral gyrus, cingulate gyrus, and prefrontal region. Psychiatry Res. 139, 127-139. doi: 10.1016/j.pscychresns.2005.05.005

Zung, W. W. (1971). A rating instrument for anxiety disorders. Psychosomatics 12, 371-379. doi: 10.1016/S0033-3182(71)71479-0
Conflict of Interest Statement: The authors declare that the research was conducted in the absence of any commercial or financial relationships that could be construed as a potential conflict of interest.

Copyright (c) $2019 \mathrm{Xu}$, Jiao, Tang, Zhang, Salvi and Teng. This is an open-access article distributed under the terms of the Creative Commons Attribution License (CC BY). The use, distribution or reproduction in other forums is permitted, provided the original author(s) and the copyright owner(s) are credited and that the original publication in this journal is cited, in accordance with accepted academic practice. No use, distribution or reproduction is permitted which does not comply with these terms. 